TRANSACTIONS OF THE

AMERICAN MATHEMATICAL SOCIETY

Volume 277, Number 2, June 1983

\title{
SPECTRAL DECOMPOSITION WITH MONOTONIC SPECTRAL RESOLVENTS
}

BY

\author{
I. ERDELYI AND WANG SHENGWANG
}

\begin{abstract}
The spectral decomposition problem of a Banach space over the complex field entails two kinds of constructive elements: (1) the open sets of the field and (2) the invariant subspaces (under a given linear operator) of the Banach space. The correlation between these two structures, in the framework of a spectral decomposition, is the spectral resolvent concept. Special properties of the spectral resolvent determine special types of spectral decompositions. In this paper, we obtain conditions for a spectral resolvent to have various monotonic properties.
\end{abstract}

1. Introduction. A spectral decomposition of a Banach space $X$, by a bounded linear operator $T: X \rightarrow X$,

(a) expresses $X$ as a finite linear sum of $T$-invariant subspaces $X_{i}$;

(b) represents $T$ as the sum of its restrictions $T_{i}=T \mid X_{i}$;

(c) localizes the spectrum $\sigma\left(T_{i}\right)$ of each $T_{i}$ in the closure of a given open set $G_{i}$, which intersects the spectrum $\sigma(T)$ of $T$.

The relationship between the invariant subspaces $X_{i}$ and the open sets $G_{i}$, formalized under the name of spectral resolvent, has been the study of some recent works $[1,2,8]$. In this paper, we investigate conditions under which the spectral resolvent possesses certain specific monotonic properties. Such conditions and subsequence properties infer the corresponding spectral decompositions.

For a bounded linear operator $T$, which maps an abstract Banach space $X$ over the complex field $\mathrm{C}$ into itself, we use the following notation: spectrum $\sigma(T)$, point spectrum $\sigma_{p}(T)$, resolvent set $\rho(T)$, the unbounded component of the resolvent set $\rho_{\infty}(T)$, and the resolvent operator $R(\cdot ; T)$. If $T$ has the single valued extension property then, for $x \in X, \sigma_{T}(x)$ denotes the local spectrum, $\rho_{T}(x)$ the local resolvent set and $x(\cdot)$ the local resolvent function.

For a subspace (closed linear manifold) $Y$ of $X, T \mid Y$ is the restriction of $T$ to $Y$ and $T / Y$ is the coinduced operator on the quotient space $X / Y$. Inv $T$ denotes the lattice of the invariant subspaces of $X$ under $T$. $T^{*}$ is the conjugate of $T$. If $A$ is a subset of $X$ then $A^{\perp}$ denotes the annihilator of $A$ in the dual space $X^{*}$. Given a set $S$, we write $\bar{S}$ for the closure, $S^{c}$ for the complement, $d(\lambda, S)$ for the distance from a point $\lambda$ to $S$, and express by $\operatorname{cov} S$, the collection of all finite open covers of $S$. (G) stands for the family of all open subsets of $\mathbf{C}$. An open set $\Delta$ is called a Cauchy

Received by the editors December 28, 1981 and, in revised form, September 28, 1982. This paper, under the title Monotonic spectral resolvents has been presented at a special session on Operator Theory of the 793rd meeting of the American Mathematical Society at Bryn Mawr, Pennsylvania, March 16-17, 1982.

1980 Mathematics Subject Classification. Primary 47B40; Secondary 47A10, 47A15. 
domain if it has a finite number of components and the boundary $\Gamma=\partial \Delta$ is a positively oriented finite system of closed, nonintersecting, rectifiable Jordan curves.

Throughout this paper $T$ is a bounded linear operator mapping the underlying Banach space $X$ into itself.

1.1. Definition. A spectral decomposition of $X$ by $T$ is a finite system $\left\{\left(G_{i}, X_{i}\right)\right\}$ $\subset$ SS $\times \operatorname{Inv} T$, satisfying the following conditions:

(i) $\left\{G_{i}\right\} \in \operatorname{cov} \sigma(T)$;

(ii) $X=\sum_{i} X_{i}$;

(iii) $\sigma\left(T \mid X_{i}\right) \subset \bar{G}_{i}$, for all $i$.

1.2. Definition [1]. A map $E:$ \& $\rightarrow \operatorname{Inv} T$ is called a spectral resolvent of $T$ if it satisfies the following conditions:

(I) $E(\varnothing)=\{0\}$;

(II) for any $\left\{G_{i}\right\} \in \operatorname{cov} \sigma(T),\left\{\left(G_{i}, E\left(G_{i}\right)\right)\right\}$ is a spectral decomposition of $X$ by $T$. Although the spectral resolvent fails to be unique, the properties they have in common characterize specific types of spectral decompositions. In this vein, we mention that an operator $T$ having a spectral resolvent possesses the single valued extension property [1] and, moreover, it is decomposable [8] in the sense of Foias [4].

The following types of invariant subspaces will be involved in our study.

1.3. Definition [5]. A subspace $Y$ of $X$ is said to be analytically invariant under $T$ if; for every function $f: D \rightarrow X$ analytic on some open $D \subset \mathbf{C}$, the condition

$$
(\lambda-T) f(\lambda) \in Y \text { on } D
$$

implies that $f(\lambda) \in Y$ on $D$.

An analytically invariant subspace is also invariant under $T[6]$.

1.4. Definition [4]. $Y \in \operatorname{Inv} T$ is said to be a spectral maximal space of $T$ if, for any $Z \in \operatorname{Inv} T$, the inclusion $\sigma(T \mid Z) \subset \sigma(T \mid Y)$ implies that $Z \subset Y$.

If $T$ has the single valued extension property then, for any set $S \subset \mathbf{C}$,

$$
X_{T}(S)=\left\{x \in X: \sigma_{T}(x) \subset S\right\}
$$

is a linear manifold in $X$. If $T$ is a decomposable operator then, for any $G \in$ SS, $\overline{X_{T}(G)}$ is an analytically invariant subspace under $T$ [5] and, for any closed $F \subset \mathbf{C}$, $X_{T}(F)$, in particular $X_{T}(\bar{G})$, is a spectral maximal space of $T$ [4]. Moreover, for a decomposable $T$, we have

$$
\overline{G \cap \sigma(T)} \subset \sigma\left[T \mid X_{T}(\bar{G})\right] \subset \bar{G} \cap \sigma(T) .
$$

1.5. Definition [9]. $Y \in \operatorname{Inv} T$ is said to be a $T$-absorbent space if, for every $y \in Y$ and all $\lambda \in \sigma(T \mid Y)$, the equation $(\lambda-T) x=y$ has all solutions $x$, if any, contained in $Y$.

If $T$ has the single valued extension property, then every $T$-absorbent space is analytically invariant under $T$.

1.6. Proposition [2]. Let $\left\{\left(G_{i}, X_{i}\right)\right\}_{i=1,2}$ be a spectral decomposition of $X$ by $T$ in terms of T-absorbent spaces $X_{1}$ and $X_{2}$. Then

$$
\sigma\left(T \mid X_{1} \cap X_{2}\right) \subset \sigma\left(T \mid X_{1}\right) \cap \sigma\left(T \mid X_{2}\right) .
$$


1.7. Proposition. If, for $X_{1}, X_{2} \in \operatorname{Inv} T, X=X_{1}+X_{2}$ then

$$
\sigma(T) \subset \sigma\left(T \mid X_{1}\right) \cup \sigma\left(T \mid X_{2}\right) \cup \sigma_{p}(T) .
$$

In particular, if $T$ has the single valued extension property, then

$$
\sigma(T) \subset \sigma\left(T \mid X_{1}\right) \cup \sigma\left(T \mid X_{2}\right) .
$$

Proof. Let $\lambda \in \rho\left(T \mid X_{1}\right) \cap \rho\left(T \mid X_{2}\right)-\sigma_{p}(T)$ and $x \in X$. There is a representation for $x, x=x_{1}+x_{2}$ with $x_{i} \in X_{i}, i=1,2$. For $y_{i}=R\left(\lambda ; T \mid X_{i}\right) x_{i}, i=1,2$, and $y=y_{1}+y_{2}$ we have

$$
(\lambda-T) y=(\lambda-T) y_{1}+(\lambda-T) y_{2}=x_{1}+x_{2}=x
$$

and hence $\lambda-T$ is surjective. Furthermore, since $\lambda \notin \sigma_{p}(T)$, we have $\lambda \in \rho(T)$. The last statement of the proposition follows from [3, Theorem 2].

Property (1.1) of $X_{T}(\cdot)$ has an interesting variant in terms of a spectral resolvent $E$, expressed by [8, Proposition 16]. For completeness, we recall that property and provide it with a shorter proof.

1.8. Proposition. If $T$ has a spectral resolvent $E$ then, for any $G \in \mathbb{S}$,

$$
\overline{G \cap \sigma(T)} \subset \sigma[T \mid E(G)] \text {. }
$$

Proof. Let $\lambda \in G \cap \sigma(T)$ be given and let $H \in(S)$ be such that $\{G, H\} \in$ $\operatorname{cov} \sigma(T)$ with $\lambda \notin \bar{H}$. Then $X=E(G)+E(H)$ and Proposition 1.7 implies

$$
\sigma(T) \subset \sigma[T \mid E(G)] \cup \sigma[T \mid E(H)] .
$$

Since $\lambda \in[G \cap \sigma(T)]-\bar{H}$, it follows from (1.4) that $\lambda \in \sigma[T \mid E(G)]$ and hence inclusion (1.3) holds.

If $T$ has a spectral resolvent $E$, then $T$ has a maximal spectral resolvent $E_{m}$ in the sense that, for every $G \in \mathbb{B S}$ and all spectral resolvents $E$ of $T$,

$$
E(G) \subset E_{m}(G)=X_{T}(\bar{G}) .
$$

Since, clearly $\overline{X_{T}(G)} \subset X_{T}(\bar{G})$, where the inclusion may be proper, some spectral resolvents $E$ may be such that

$$
\overline{X_{T}(G)} \subset E(G) \subset X_{T}(\bar{G}) \text { for all } G \in \mathbb{B S} .
$$

Condition (1.5) endows $E$ with some remarkable properties, which will be the topic of the following sections.

\section{Monotonic spectral resolvents.}

2.1. Definition. A spectral resolvent $E$ is said to be monotonic if $G_{1}, G_{2} \in \mathbb{B S}$ and $\bar{G}_{1} \subset G_{2}$ imply that $E\left(G_{1}\right) \subset E\left(G_{2}\right)$.

Note that (1.5) is a sufficient condition for a spectral resolvent $E$ of $T$ to be monotonic. In fact, if the open sets $G_{1}, G_{2}$ are such that $\bar{G}_{1} \subset G_{2}$, then (1.5) implies the inclusions

$$
E\left(G_{1}\right) \subset X_{T}\left(\bar{G}_{1}\right) \subset \overline{X_{T}\left(G_{2}\right)} \subset E\left(G_{2}\right) .
$$


2.2. TheOREM. Let $T$ have a spectral resolvent $E$. If for any pair $G_{1}, G_{2} \in \mathbb{S}, E$ satisfies condition

$$
\sigma\left[T \mid E\left(G_{1}\right) \cap E\left(G_{2}\right)\right] \subset \bar{G}_{1} \cap \bar{G}_{2}
$$

then property (1.5) holds and $E$ is monotonic.

Proof. Given $G_{1} \in \mathbb{S}$, let $x \in X_{T}\left(G_{1}\right)$. Choose $G_{2} \in$ SS such that $\left\{G_{1}, G_{2}\right\} \in$ $\operatorname{cov} \sigma(T)$ and $\sigma_{T}(x) \cap \bar{G}_{2}=\varnothing$ (this is possible because $\sigma_{T}(x)$ is closed and is contained in $G_{1}$ ). To avoid repetitions, we divide the remainder of the proof in two parts.

Part A. There is a representation of $x$,

$$
x=x_{1}+x_{2} \text { with } x_{i} \in E\left(G_{i}\right), i=1,2 .
$$

In view of some elementary properties, the local spectra of $x_{1}$ and $x_{2}$ are contained in some pertinent sets

$$
\sigma_{T}\left(x_{1}\right) \subset \sigma_{T}(x) \cup\left(\bar{G}_{1} \cap \bar{G}_{2}\right), \quad \sigma_{T}\left(x_{2}\right) \subset \bar{G}_{1} \cap \bar{G}_{2} .
$$

For $\lambda \in \rho_{T}(x) \cap\left(\bar{G}_{1} \cap \bar{G}_{2}\right)^{c}=H$, we have $x(\lambda)=x_{1}(\lambda)+x_{2}(\lambda)$. Let $\Delta$ be a Cauchy domain with boundary $\Gamma$ such that $\sigma_{T}(x) \subset \Delta$ and $\bar{\Delta} \subset\left(\bar{G}_{1} \cap \bar{G}_{2}\right)^{c}$. The functional calculus gives

$$
x=\frac{1}{2 \pi i} \int_{\Gamma} x(\lambda) d \lambda=\frac{1}{2 \pi i} \int_{\Gamma} x_{1}(\lambda) d \lambda .
$$

For every $\lambda_{0} \in \Gamma$, there is a neighborhood $V \subset H$ of $\lambda_{0}$ and there are functions $f_{i}$ : $V \rightarrow E\left(G_{i}\right)(i=1,2)$ analytic on $V$ such that

$$
x_{1}(\lambda)=f_{1}(\lambda)+f_{2}(\lambda) \text { on } V .
$$

It follows from

$$
(\lambda-T) x_{1}(\lambda)=x_{1} \text { on } \rho_{T}\left(x_{1}\right),
$$

that the function $g: V \rightarrow E\left(G_{1}\right) \cap E\left(G_{2}\right)$ defined by

$$
g(\lambda)=x_{1}-(\lambda-T) f_{1}(\lambda)=(\lambda-T) f_{2}(\lambda)
$$

is analytic on $V$.

Part B. Since $V \subset\left(\bar{G}_{1} \cap \bar{G}_{2}\right)^{c} \subset \rho\left[T \mid E\left(G_{1}\right) \cap E\left(G_{2}\right)\right]$, the function $h: V \rightarrow$ $E\left(G_{1}\right) \cap E\left(G_{2}\right)$ defined by

$$
h(\lambda)=R\left[\lambda ; T \mid E\left(G_{1}\right) \cap E\left(G_{2}\right)\right] g(\lambda)
$$

is analytic on $V$. We have

$$
(\lambda-T) h(\lambda)=g(\lambda)=(\lambda-T) f_{2}(\lambda) \text { on } V
$$

and hence the single valued extension property of $T$ implies that

$$
f_{2}(\lambda)=h(\lambda) \in E\left(G_{1}\right) \cap E\left(G_{2}\right) \subset E\left(G_{1}\right) \text { on } V .
$$

Thus, by (2.4) $x_{1}(\lambda) \in E\left(G_{1}\right)$ on $V$ and, in particular, $x_{1}\left(\lambda_{0}\right) \in E\left(G_{1}\right)$. Since $\lambda_{0}$ is arbitrary on $\Gamma$, it follows from (2.3) that $x \in E\left(G_{1}\right)$. Thus, $X_{T}\left(G_{1}\right) \subset E\left(G_{1}\right)$ and this establishes (1.5). Consequently, $E$ is a monotonic spectral resolvent. 
2.3. CoROllary. Let $E$ be a spectral resolvent of $T$. If for each $G \in \mathbb{G}$, any one of the following conditions holds, then $E$ is monotonic.

(1) $\sigma\left[T^{*} \mid E(G)^{\perp}\right] \subset G^{c}$;

(2) $\sigma[T / E(G)] \subset G^{c}$;

(3) $E(G)$ is analytically invariant;

(4) $E(G)$ is T-absorbent.

Proof. Conditions (1)-(3) are equivalent [1]. Moreover, since $T$ has the single valued extension property, every $T$-absorbent space is analytically invariant under $T$. Thus, it suffices to prove the statement of the corollary under hypothesis (4). Given $G_{1}, G_{2} \in$ SS, Proposition 1.6 implies

$$
\sigma\left[T \mid E\left(G_{1}\right) \cap E\left(G_{2}\right)\right] \subset \sigma\left[T \mid E\left(G_{1}\right)\right] \cap \sigma\left[T \mid E\left(G_{2}\right)\right] \subset \bar{G}_{1} \cap \bar{G}_{2} .
$$

Now, Theorem 2.2 concludes the proof.

2.4. Corollary. Let $T$ have a spectral resolvent $E$. If $\sigma(T)$ has empty interior and $\rho_{\infty}(T)=\rho(T)$ (in particular, if $\sigma(T)$ is contained on an open Jordan curve), then $E$ is monotonic.

Proof. It suffices to show that for every $G \in \mathbb{S}, E(G)$ is analytically invariant under $T$. Let $f: D \rightarrow X$ be analytic on an open $D \subset \mathbf{C}$ such that for every $G \in$ (S),

$$
(\lambda-T) f(\lambda) \in E(G) \text { on } D \text {. }
$$

Since $\sigma(T)$ has empty interior, $D-\sigma(T)$ is a nonempty open set. Then, since $\rho_{\infty}(T)=\rho(T)$, we have

$$
f(\lambda)=R(\lambda ; T)(\lambda-T) f(\lambda) \in E(G) \text { for all } \lambda \in D-\sigma(T)
$$

and $f(\lambda) \in E(G)$ on $D$, by analytic continuation.

As a summary of this section, the "spectral inclusion property" (1.5) and the "spectral invariance property" (2.1) proved to be sufficient conditions for a spectral resolvent $E$ to be monotonic. By strengthening the monotonic spectral resolvent concept, (1.5) is heightened to a necessary and sufficient condition for the validity of the new monotonic attribute of a spectral resolvent.

\section{Strongly monotonic spectral resolvents.}

3.1. Definition. A spectral resolvent $E$ is said to be strongly monotonic if $G, G_{1}, G_{2} \in \mathscr{S}$ and $\bar{G}_{1} \cap \bar{G}_{2} \subset G$ imply $E\left(G_{1}\right) \cap E\left(G_{2}\right) \subset E(G)$.

Evidently, every strongly monotonic spectral resolvent is monotonic. As an example, if $T$ has a spectral resolvent $E$ then its maximal spectral resolvent $E_{m}$ is strongly monotonic. Indeed, $G, G_{1}, G_{2} \in \mathbb{S}$ and $\bar{G}_{1} \cap \bar{G}_{2} \subset G$ imply

$$
E_{m}\left(G_{1}\right) \cap E_{m}\left(G_{2}\right)=X_{T}\left(\bar{G}_{1}\right) \cap X_{T}\left(\bar{G}_{2}\right)=X_{T}\left(\bar{G}_{1} \cap \bar{G}_{2}\right) \subset X_{T}(\bar{G})=E_{m}(G) .
$$

3.2. THEOREM. Let $E$ be a spectral resolvent of $T$. E is strongly monotonic if and only if (1.5) holds for every $G \in \mathbb{B S}$.

Proof. We only have to prove the "only if" part. Assume that $E$ is strongly monotonic. Given $G \in \mathbb{S}$, let $x \in X_{T}(G)$. Let $\left\{G_{1}, G_{2}\right\} \in \operatorname{cov} \sigma(T)$ be such that

$$
\sigma_{T}(x) \subset G_{1} \subset \bar{G} \subset G \text { and } \sigma_{T}(x) \cap \bar{G}_{2}=\varnothing .
$$


Follow verbatim Part A of the proof of Theorem 2.2. Let $K \in \mathbb{S}$ be such that

$$
\bar{G}_{1} \cap \bar{G}_{2} \subset K \subset \bar{K} \subset G, \quad \bar{K} \cap \sigma_{T}(x)=\varnothing \quad \text { and } \quad V \cap \bar{K}=\varnothing .
$$

$E$ being strongly monotonic, we have $g(\lambda) \in E(K)$ on $V$. The function $h: V \rightarrow E(K)$ defined by $h(\lambda)=R[\lambda ; T \mid E(K)] g(\lambda)$ is analytic on $V$ and

$$
(\lambda-T) h(\lambda)=(\lambda-T) f_{2}(\lambda) \text { on } V .
$$

By the single valued extension property of $T$,

$$
f_{2}(\lambda)=h(\lambda) \in E(K) \text { on } V .
$$

$E$ being monotonic, we have

$$
x_{1}(\lambda) \in E\left(G_{1}\right)+E(K) \subset E(G) \text { on } V
$$

and, in particular, $x_{1}\left(\lambda_{0}\right) \in E(G)$. Since $\lambda_{0}$ is arbitrary on $\Gamma$, it follows from (2.3) that $x \in E(G)$. Since $x$ is arbitrary in $X_{T}(G)$, the proof concludes with $\overline{X_{T}(G)} \subset$ $E(G)$.

Another characterization of a strongly monotonic spectral resolvent involves the range of the local resolvent function.

3.3. THEOREM. Let $E$ be a spectral resolvent of $T$. The following assertions are equivalent:

(i) $E$ is strongly monotonic;

(ii) $G_{1}, G_{2} \in$ SS, $\bar{G}_{1} \subset G_{2}$ and $x \in E\left(G_{1}\right)$ imply $\left\{x(\lambda): \lambda \in \rho_{T}(x)\right\} \subset E\left(G_{2}\right)$.

Proof. (i) $\Rightarrow$ (ii): Let $G_{1}, G_{2} \in$ SS be such that $\bar{G}_{1} \subset G_{2}$. By Theorem 3.2, we have

$$
E\left(G_{1}\right) \subset X_{T}\left(\bar{G}_{1}\right) \subset X_{T}\left(G_{2}\right) \subset E\left(G_{2}\right) .
$$

Let $x \in E\left(G_{1}\right)$ be given. Then $x \in X_{T}\left(\bar{G}_{1}\right)$ and since $X_{T}\left(\bar{G}_{1}\right)$ is a spectral maximal space of $T,(3.1)$ implies

$$
\left\{x(\lambda): \lambda \in \rho_{T}(x)\right\} \subset X_{T}\left(\bar{G}_{1}\right) \subset E\left(G_{2}\right) .
$$

(ii) $\Rightarrow$ (i): Let $G \subset \mathbf{C}$ be an open set and let $x \in X_{T}(G)$. Choose $G_{1} \in \mathbb{B S}$ such that $\sigma_{T}(x) \subset G_{1} \subset \bar{G}_{1} \subset G$. Let $G_{2} \in$ (S) satisfy conditions

$$
\sigma(T) \subset G_{1} \cup G_{2}, \quad \sigma_{T}(x) \cap \bar{G}_{2}=\varnothing .
$$

Then $x$ has a representation $x=x_{1}+x_{2}$ with $x_{i} \in E\left(G_{i}\right), i=1,2$. As obtained in an earlier proof, we have (2.2)

$$
\sigma_{T}\left(x_{1}\right) \subset \sigma_{T}(x) \cup\left(\bar{G}_{1} \cap \bar{G}_{2}\right), \quad \sigma_{T}\left(x_{2}\right) \subset \bar{G}_{1} \cap \bar{G}_{2} .
$$

Let $\Delta$ be a Cauchy domain with boundary $\Gamma \subset \rho_{T}(x) \cap\left(\bar{G}_{1} \cap \bar{G}_{2}\right)^{c}$, such that $\sigma_{T}(x) \subset \Delta$ and $\bar{\Delta} \cap\left(\bar{G}_{1} \cap \bar{G}_{2}\right)=\varnothing$. Then

$$
x=\frac{1}{2 \pi i} \int_{\Gamma} x(\lambda) d \lambda=\frac{1}{2 \pi i} \int_{\Gamma} x_{1}(\lambda) d \lambda .
$$

Since $x_{1} \in E\left(G_{1}\right)$ and $\bar{G}_{1} \subset G$, hypothesis (ii) implies

$$
\left\{x_{1}(\lambda): \lambda \in \rho_{T}(x)\right\} \subset E(G) .
$$

Then, by (3.2), $x \in E(G)$ and hence $X_{T}(G) \subset E(G)$. Now, Theorem 3.2 concludes the proof. 
A further characterization of a strongly monotonic spectral resolvent can be obtained in terms of a localization property of the spectral resolvent. The following definition generalizes the concept of "almost localized spectrum" [10].

3.4. Definition. A spectral resolvent $E$ is said to be almost localized if $G, G_{1}, G_{2}$ $\in(S)$ and $\bar{G} \subset G_{1} \cup G_{2}$ imply $E(G) \subset E\left(G_{1}\right)+E\left(G_{2}\right)$.

The following result is due to Radjabalipour [7].

3.5. Proposition. If $T$ is decomposable then, for every closed set $F$ and $\left\{H_{1}, H_{2}\right\} \in$ $\operatorname{cov} F$, the following inclusion holds:

$$
X_{T}(F) \subset X_{T}\left(\bar{H}_{1}\right)+X_{T}\left(\bar{H}_{2}\right)
$$

Since, for every open cover $\left\{H_{1}, H_{2}\right\}$ of $F$, there is $\left\{G_{1}, G_{2}\right\} \in \operatorname{cov} F$ with $\bar{H}_{1} \subset G_{1}$ and $\bar{H}_{2} \subset G_{2}$, property (3.3) can be expressed as

$$
X_{T}(F) \subset \overline{X_{T}\left(G_{1}\right)}+\overline{X_{T}\left(G_{2}\right)} .
$$

3.6. THEOREM. Let $T$ have a spectral resolvent $E$. Then $E$ is strongly monotonic if and only if $E$ is almost localized.

Proof. In view of Theorem 3.2, we have to show that the following conditions are equivalent:

(i) $\overline{X_{T}(G)} \subset E(G)$ for all $G \in$ (S);

(ii) $G, G_{1}, G_{2} \in$ SS and $\bar{G} \subset G_{1} \cup G_{2}$ imply $E(G) \subset E\left(G_{1}\right)+E\left(G_{2}\right)$.

(i) $\Rightarrow$ (ii): Let $G, G_{1}, G_{2} \in$ (S) be such that $\bar{G} \subset G_{1} \cup G_{2}$. Since $T$ is decomposable, (3.4) implies

$$
E(G) \subset X_{T}(\bar{G}) \subset \overline{X_{T}\left(G_{1}\right)}+\overline{X_{T}\left(G_{2}\right)} \subset E\left(G_{1}\right)+E\left(G_{2}\right) .
$$

(ii) $\Rightarrow$ (i): Given $G \in(S)$, let $x \in X_{T}(G)$. Further, let $H_{0}$ be a relatively compact, open neighborhood of $\sigma(T)$. Then

$$
x \in X=E\left(H_{0}\right) \text { and } \sigma_{T}(x) \subset \sigma(T) \subset H_{0} .
$$

Let $\varepsilon$ be arbitrary, with $0<\varepsilon<\sup _{\lambda \in \partial H_{0}} d\left[\lambda, \sigma_{T}(x)\right]$. Define the open sets

$$
H=\left\{\lambda \in \mathbf{C}: d\left[\lambda, \sigma_{T}(x)\right]<\varepsilon\right\}, \quad H^{\prime}=\left\{\lambda \in \mathbf{C}: d\left(\lambda, H_{0}\right)<\frac{\varepsilon}{6}\right\} .
$$

For every $\lambda \in \bar{H}^{\prime} \cap H^{c}$, let $D_{\lambda}=\{\mu \in \mathbf{C}:|\mu-\lambda|<\varepsilon / 3\}$. Then $\left\{D_{\lambda}: \lambda \in \bar{H}^{\prime} \cap H^{c}\right\}$ is an open cover of $\bar{H}^{\prime} \cap H^{c}$. Since $\bar{H}^{\prime} \cap H^{c}$ is compact, there is a finite collection $\left\{\lambda_{1}, \lambda_{2}, \ldots, \lambda_{n}\right\} \subset \overline{H^{\prime}} \cap H^{c}$ such that

$$
\bar{H}^{\prime} \cap H^{c} \subset \bigcup_{i=1}^{n} D_{i}, \quad \text { where } D_{i}=D_{\lambda} \text { for } \lambda=\lambda_{i} .
$$

For $1 \leqslant i \leqslant n$, define

$$
K_{i}=\left\{\mu \in \mathbf{C}:\left|\mu-\lambda_{i}\right|<\frac{2}{3} \varepsilon\right\}, \quad \Delta_{i}=\left\{\mu \in C:\left|\mu-\lambda_{i}\right|<\frac{\varepsilon}{2}\right\} .
$$

Clearly, $\bar{K}_{i} \cap \sigma_{T}(x)=\varnothing, 1 \leqslant i \leqslant n$. Put

$$
H_{1}=\left\{\lambda \in \mathbf{C}: d\left(\lambda, H_{0}\right)<\frac{\varepsilon}{9 n}\right\}-\bar{\Delta}_{1} .
$$


It is easy to see that $\bar{H}_{1} \cap \bar{D}_{1}=\varnothing$. Since

$$
\bar{H}_{0} \subset H_{1} \cup \bar{\Delta}_{1} \subset H_{1} \cup K_{1} \text {, }
$$

we have

$$
x \in E\left(H_{0}\right) \subset E\left(H_{1}\right)+E\left(K_{1}\right) .
$$

For $G_{1}=H_{1}, G_{2}=K_{1}$, follow Part A of the proof of Theorem 2.2. Note that the boundary $\Gamma$ of the Cauchy domain $\Delta$ in Part A, verifies inclusions

$$
\Gamma \subset \rho_{\infty}\left[T \mid E\left(K_{1}\right)\right] \subset \rho\left[T \mid E\left(H_{1}\right) \cap E\left(K_{1}\right)\right] .
$$

The function $h: V \rightarrow E\left(H_{1}\right) \cap E\left(K_{1}\right)$, defined by

$$
h(\lambda)=R\left[\lambda ; T \mid E\left(H_{1}\right) \cap E\left(K_{1}\right)\right] g(\lambda)
$$

verifies equality

$$
(\lambda-T) h(\lambda)=(\lambda-T) f_{2}(\lambda) \text { on } V
$$

which implies

$$
f_{2}(\lambda)=h(\lambda) \in E\left(H_{1}\right) \cap E\left(K_{1}\right) \text { on } V .
$$

Thus, with reference to Part A, (2.4) implies that $x_{1}(\lambda) \in E\left(H_{1}\right)$ on $V$, and hence $x_{1}\left(\lambda_{0}\right) \in E\left(H_{1}\right) . \lambda_{0} \in \Gamma$ being arbitrary, $x \in E\left(H_{1}\right)$ by $(2.3)$.

Inductively, define

$$
H_{k}=\left\{\lambda \in \mathbf{C}: d\left(\lambda, H_{k-1}\right)<\varepsilon / 9 n\right\}-\bar{\Delta}_{k}, \quad 1 \leqslant k \leqslant n .
$$

Then $\left\{H_{k}, K_{k}\right\}$ covers $\bar{H}_{k-1}$ and $\bar{H}_{k} \cap \bar{D}_{i}=\varnothing, 1 \leqslant i \leqslant k$. In view of hypothesis (ii), $E\left(H_{k-1}\right) \subset E\left(H_{k}\right)+E\left(K_{k}\right)$, and the hypothesis $x \in E\left(H_{k-1}\right)$ of the induction gives $x \in E\left(H_{k}\right)+E\left(K_{k}\right)$. As for $k=1$, by using Part A of the proof of Theorem 2.2 and a conveniently defined function $h: V \rightarrow E\left(H_{k}\right) \cap E\left(K_{k}\right)$, we obtain $x \in$ $E\left(H_{k}\right)$. Thus, by the inductive process, we obtain an open set $H_{n}$ with the properties

$$
x \in E\left(H_{n}\right) \text { and } \bar{H}_{n} \subset H^{\prime}-\left(\bigcup_{i=1}^{n} \bar{D}_{i}\right) \subset H .
$$

$E$ being monotonic, $E\left(H_{n}\right) \subset E(H)$ and hence $x \in E(H)$. Since $\varepsilon$ is arbitrarily small, we may choose it such that $\bar{H} \subset G$. Then $E(H) \subset E(G)$ and hence $x \in E(G)$. Since $x \in X_{T}(G)$ is arbitrary, we obtain $\overline{X_{T}(G)} \subset E(G)$.

\section{REFERENCES}

1. I. Erdelyi, Spectral resolvents, Operator Theory and Functional Analysis, Research Notes in Math., no. 38, Pitman Advanced Publishing Program, San Francisco, London, Melbourne, 1979, pp. 51-70.

2. __ Monotonic properties of some spectral resolvents, Libertas Math. 1 (1981), 117-148.

3. J. K. Finch, The single valued extension property on a Banach space, Pacific J. Math. 58 (1975), 61-69.

4. C. Foiaş, Spectral maximal spaces and decomposable operators in Banach spaces, Arch. Math. (Basel) 14 (1963), 341-349.

5. S. Frunză, The single-valued extension property for coinduced operators, Rev. Roumaine Math. Pures Appl. 18 (1973), 1061-1065.

6. R. Lange, Strongly analytic subspaces, Operator Theory and Functional Analysis, Research Notes in Math., no. 38, Pitman Advanced Publishing Program, San Francisco, London, Melbourne, 1979, pp. $16-30$. 
7. M. Radjabalipour, Equivalence of decomposable and 2-decomposable operators, Pacific J. Math. 77 (1978), 243-247.

8. G. W. Shulberg, Spectral resolvents and decomposable operators, Operator Theory and Functional Analysis, Research Notes in Math., no. 38, Pitman Advanced Publishing Program, San Francisco, London, Melbourne, 1979, pp. 71-84.

9. F. H. Vasilescu, Residually decomposable operators in Banach spaces, Tôhoku Math. J. 21 (1969), 509-522.

10. , On the residual decomposability in dual spaces, Rev. Roumaine Math. Pures Appl. 16 (1971), 1573-1578.

Department of Mathematics, Temple University, Philadelphia, Pennsylvania 19122

Department of Mathematics, Nanjing University, Nanjing, China 